\title{
Vorwort von Verlag und Beirat
}

Die Studienbuchreihe „Seminar Geschichte“ soll den Benutzern StudentInnen und DozentInnen der Geschichtswissenschaft, aber auch VertreterInnen benachbarter Disziplinen - ein Instrument bieten, mit dem sie sich den Gegenstand des jeweiligen Bandes schnell und selbstständig erschließen können. Die Themen reichen von der Antike bis in die Gegenwart; unter Einbeziehung historischer Debatten sowie wichtiger Forschungskontroversen vermitteln die Bände konzise das relevante Basiswissen zum jeweiligen Thema.

„Seminar Geschichte“ wurde von De Gruyter Oldenbourg gemeinsam mit FachhistorikerInnen und Geschichtsdidaktikern entwickelt. Die Reihe trägt den Bedürfnissen von StudentInnen in den neuen, modularisierten und kompetenzorientierten Studiengängen Rechnung. Dabei liegt der Akzent auf der Vermittlung von aktuellen Methoden und Ansätzen. Im Sinne einer möglichst effizienten akademischen Lehre sind die Bände stark quellenbasiert und nach fachdidaktischen Gesichtspunkten strukturiert. Sie stellen nicht nur den gegenwärtigen Kenntnisstand zu ihrem Thema dar, sondern führen über die intensive Auseinandersetzung mit maßgeblichen Quellen zudem fundiert in geschichtswissenschaftliche Fragestellungen und Methoden ein. Dabei steht die Problemorientierung im Vordergrund. Unabdingbar ist dafür, dass die Quellen nicht abschließend ausgedeutet werden, sondern eine Grundlage für die eigene Erschließung und Bearbeitung bilden. Hierzu enthält jeder Band kommentierte Lektüreempfehlungen, Fragen zum Textverständnis und zur Vertiefung sowie Anregungen zur Weiterarbeit.

Jeder Band stellt eine autonome Einheit dar. Wichtige Quellen sind im Band enthalten, damit sie nicht mitgeführt oder online aufgerufen werden müssen; zentrale Fachbegriffe werden im Glossar im Anhang erklärt. Ergänzend findet sich auf der Website des Verlages zu jedem Band der Reihe zusätzliches Material (z.B. weitere und/oder originalsprachliche Quellen, thematisch relevante Abbildungen, weiterführende Links oder zusätzliche vertiefende und zur Weiterarbeit anregende Fragen; für den vorliegenden Band: www.degruyter.com/view/product/465998). Passagen, 
für die zur Vertiefung weiteres Material bereitsteht, sind durch das nebenstehende Symbol hervorgehoben.

Durch seinen modularen Aufbau macht jeder Band auch ein Angebot für ein Veranstaltungsmodell bzw. eröffnet die Möglichkeit, einzelne Kapitel als Grundlage für Lehreinheiten zu nehmen. Der Aufbau in 14 Kapiteln spiegelt die (in der Regel) 14 Lehreinheiten eines Semesters und unterstreicht den Anspruch, das zu vermitteln, was innerhalb eines Semesters gut gelehrt und gelernt werden kann. Der einheitliche Aufbau aller Bände der Reihe sorgt für konzeptionelle Übersichtlichkeit und Verlässlichkeit in der Benutzung: Er bietet StudentInnen und DozentInnen eine gemeinsame Grundlage, um sich neue Themenfelder zu erschießen. 